\title{
The Ideal Resident Doctor: A Resident's Perspective
}

\author{
Dr. O. A.Omisanjo MBBS (lb) \\ Department of Surgery, University, College Hospital, PMB 5116, \\ Ibadan, Oyo State, Nigeria.
}

The Hippocratic oath, the oath to which all doctors-including residents-swear at the beginning of their respective medical careers, gives one an insight as to what is required of a doctor as regards his duty to his patient, his teacher and his student. The residency period is a peculiar time in a doctor's career during which he assumes many roles at the same time-a doctor of his patients, a student of his trainers and a teacher of medical students.

$\mathrm{He}$ is also training to practice as a professional in an increasingly competitive environment which continues to get even smaller by the unavoidable consequences of globalization.

In examining who an ideal resident is, it is imperative to consider the kind of person he should be and what he should know or know how to do. It is however also pertinent to consider the totality of the environment in which the ideal resident doctor must work. This is even more so considering the radical change in healthcare delivery that promotes the role of the physician as clinical and economic manager rather than professional decision-maker and patient advocate in this era of managed medical care.

The Hippocratic oath suggests that the resident should treat his trainer as his parent. The scope of the resident-trainer relationship can only then be imagined. The same document enjoins a resident indeed like any other doctor "to apply, for the benefit of the sick, all measures which are required" and

All Correspondence to Dr. O.A. Omisanjo

Department of Surgery,

University College Hospital, PMB 51 16, Ibadan,

Oyo State, Nigeria.

E.Mail: sanjofunmi@yahoo.com to "keep them from harm and injustice"[1]

The scope of what has to be done to fulfill the two aforementioned responsibilities will depend on the peculiarities and the vagaries of the resident's conditions and his environment. Indeed requirements like these lend themselves to personal and cultural interpretations.

Can one surmise that the attributes of an ideal resident should be as suggested by the Hippocratic Oath? Can one say that a resident doctor who enjoys a near-patriarchal relationship with his trainer, one who does all that is needed for the good of his patient, learns all that is required of him and is himself a great teacher fits the bill of an ideal resident doctor? I think that will be a rather simplistic approach. Indeed a growing number of physicians have come to feel that the Hippocratic Oath is inadequate to address the realities of a medical world that has witnessed huge scientific, economic, political and social changes.

Traditionally most training programmes reinforce the primacy of what a resident should know over subjective experience underlining the expectation that an ideal resident is one who knows the much that is required of his specialty in terms of skills and knowledge-teaching, research, etc. [2] Indeed over the years a lot of emphasis has been placed on the need for residents to acquire adequate knowledge required for specialist practice. A resident is expected to have cutting edge information about his specialty. This is not surprising considering that the period of residency is essentially the last phase of a structured training for the specialist. The acquisition of this kind of cutting edge information 
naturally calls for a robust interaction with other colleagues under peer controlled learning programmes and regular institutional academic meetings.

As important as the need for a thorough scientific background is for a resident, the present atmosphere in most training programmes tends to make the acquisition of such knowledge by the resident a near impossible task. This need for the acquisition of sound knowledge is often overridden by the service needs of the training institutions. While the provision of high quality care and service is an unavoidable and indeed integral part of the training of a resident, ideally a critical balance must be struck such that the resident still has adequate time for more didactic academic pursuits.

Recently, there has been an increasing clamour for the practice of Evidence based Medicine. Evidence based medicine is "the process of systematically finding, appraising, and using contemporaneous research findings as the basis for the clinical decisions"[3].An ideal resident's clinical options should not be limited to age long institutional practices some of which are based on anecdotal experiences of past authorities in the specialty. In managing patients, he needs to demonstrate a good grasp of "alternative options" of proven efficacy. All of this also calls for a regular sharing of ideas and experiences with colleagues from other centres in conferences, symposia, workshops, et cetera.

It can always be argued that a resident ideally is training to acquire enough skills to positively impact on his own immediate environment and thus the scope of what he should know and know how to do should be driven by the needs of his environment and the facilities available for him to work with However, in a world faced with increasing demand for globalization and a relatively fluid transcontinental migration of professionals, this line of argument is then fraught with a number of fallacies. The ideal seems to be that irrespective of where a resident trains, he needs to acquire a prerequisite level of expertise that affords him the opportunity of a good standing in the international arena. When training centres are lacking in the needed expertise and technological back up, hen efforts must be made to complement the resident's training through a well planned exchange programme with better equipped centres. It is intriguing that some centres in the West with state of the art equipment still realise the need for their resident to acquire some "third world relevant" experience and have recommended that their trainees spend some time in third world countries. Centres in the third world ought to take a cue from this.

Today's resident is not only a clinician but also a researcher. Every resident must have the opportunity to engage in research work in which he seeks to unravel the medical puzzles of his time. Such work need not be left to scientists dedicated to research work alone. The discipline learnt thereby can of course complement the resident's approach to clinical practice.

A doctor is generally regarded as the leader of the medical team even though there are people who may contest this. The transition from a resident to a consultant with its attending responsibilities is often abrupt and the challenges may thus be daunting. It is imperative that a resident inculcates the needed managerial and leadership traits while still training.

Beyond what an ideal resident should know, we need to take a critical look at the kind of person he should be as a trainee, a doctor and a trainer himself. The basic requirement of a trainee to his boss is traditionally regarded as loyalty-to do his boss's bidding and as Hippocrates said "treat he who taught me this art as a parent' [1]. Indeed, some have likened residency training to an apprenticeship of some sort. The scope of this type of relationship is too broad and culture related for one to start itemizing the various desirable traits in such a relationship. What must be stressed, though, is that beyond having a trainer, the trainee needs to see a "mentor figure" in his boss-one who as it were shows him the way through the training programme with genuine interest. Today's resident must not be under a "master-servant" relationship. Naturally, it is expected that a resident should then give his own trainees (medical students and interns) as much mentoring as he would desire of his boss.

The ideal resident is the kind of doctor that does all that is needed for the good of his patient. It 
is however important to note that he does not carry out his duty perfunctorily. The resident indeed like any other doctor needs to show compassion in all his dealings with the patient. Some have argued that this may not always be possible [4] but he must always try. Moreover simple gestures of kindness sometimes make a greater impact on patients than any display of exceptional brilliance [5]. Most patients want to know how much we care and not how much we know. A resident must be humble enough to admit his limitations and avoid that ever tempting air of "I know it all" that most doctors unconsciously demonstrate in treating patients. He should be able to forgive himself and others. These humanistic attributes though agreed by most to be vital are often lacking in the doctor-patient relationship. Though we can further itemize several of the desirable traits of the ideal resident for example his dressing code and perhaps how much he should earn, most of it will be summed up in the need for humanism and professionalism.

"While traditional residence training approaches may not preclude residents' development of humanistic and professional values, the lack of explicit attention to these aspects of training often results in these values being neglected or subtly devalued'[2]

Beyond whatever may be the prerequisite skills of a resident doctor in terms of his role as a traineer, a care giver and a teacher himself, it is also important that the resident doctor has humanistic and professional values. 'The Doctor'-a painting by Fildes [6]-which was widely reproduced by the American Medical Association(AMA) during the middle years of the twentieth century reveals a number of important themes about the desirable humanistic traits of an ideal physician. Humility; compassion, forgiveness, integrity and respect are projected as being important. Indeed the American Board of Internal Medicine(ABIM) now requires residency programmes to evaluate trainees' humanistic behaviours including the qualities of integrity, respect and compassion[2].

What Are the Requirements of an Ideal Resident Doctor as a Professional?

According to the American Board of Internal Medicine, professionalism "comprises those attitudes and behaviours that sustain the interests of the patient above one's own self-interest. Professionalism entails altruism, accountability, commitment to excellence, duty and commitment to service, honour and respect for others." [2]: Humanism and professionalism are both however inextricably woven into the art and practice of medicine.

Beyond the universally accepted characteristics of honesty, integrity, altruism, honour and duty, physicians must acquire other attributes if they are to be effective, caring, and satisfied professionals in a progressively more complicated health care system with its competing demands on time and resources, advancing technology and increasing challenges to the physician-patient relationship. These other attributes include a commitment to lifelong and self-directed learning, a willingness to work collaboratively with patients and colleagues, and an interest in and respect for the subjective experiences of others[2]

While it is important for a resident to demonstrate these traits or at least strive to acquire them, it is important to note that this will only be possible in a learner-centered environment that supports the acquisition of professional and humanistic values desirable for an ideal resident doctor. Many residency training programmes tend to brutalize residents and seem to assume that doctors can rise above their own physiology. Yet there is no objective basis to suggest that doctors are in any way different from other professionals in terms of coping with stress [7]

Indeed "recent evidence suggests that this period (of residency) is associated with significant depression, anger, cynicism and emotional withdrawal, and there are concerns about its effect on the attitudes and future of physicians" [7].Indeed "sleep deprivation and fatigue in residents jeopardize patient safety and place residents at risk for motor vehicle accidents"[8]. Physical exhaustion, irresponsible behaviour and sleep disorders consequent on stress have previously been documented amongst surgical resident doctors in a Nigerian teaching hospital.[9].

One is stressing the importance of what the work environment of the ideal resident doctor should be because "many programmes continue such de 
humanizing policies as extremely long working hours and traditional hierarchical work environments in which junior members are openly humiliated or shown disrespect" [2] .Ndom and Makanjoula have also noted that a poor work environment was a stressor in residency training in another tertiary health care centre in Nigeria [10]. In addition, a recent broad sampling of second year residents revealed both a high incidence of perceived mistreatment and that positive learning experiences and lack of mistreatment enhanced satisfaction during training[2].

Meanwhile the belief of Markakis et al is that trainees(residents) when treated with respect, caring and acknowledgement of their individual needs and strengths, will in turn communicate with and treat their patients in a respectful, caring manner [2].

The above is not meant to excuse a resident from his obligation to inculcate the aforementioned desirable traits of an idea resident.Afterall one cannot say (paraphrasing the words of Smith DG and Newton L in "Physician and Patient: respect for mutuality") that "you could put a white coat on Hitler, and if you could only get him to follow the ethical rules and make his environment conducive, he would be an all-right doctor!"'[11] But the converse is also not true because a supposed ideal resident with all the aforementioned positive traits working in a dehumanizing environment is unlikely to turn out well. Indeed, "for many residents, fatigue cultivates anger, resentment, and bitterness rather than kindness, compassion, or empathy" [12]. Such is the strength of the interplay between the resident's work environment and his own manifested characteristics.

All residents must strive to be the ideal, but so also must genuine attempts be made by the relevant authorities and institutions to ensure that resident's working conditions are optimal if not ideal. Some recent articles published in Nigeria on different aspects of postgraduate professional medical training have suggested that stakeholders in postgraduate medical education should do more in making the lot of residents better $[9,10,13]$.

It was once said of Sir William Osler-the great physician whose name is still being invoked in modern day discussions in medicine- that "his time was ripe for him, and he was ripe for his time"[14].
Residents must always make themselves "ripe for their time" whatever it takes. Equally important it is however that the relevant authorities also make time "ripe for residents". While the ideal is oftentimes unattainable, we must all at least be seen to have done our part.

The ideal scenario will then be that of a resident with the desirable traits working "in a healthy environment where honesty, respect, collaboration, and accountability are seen as core values and are practiced institutionally. In other words, a consistent culture in which the same values and behaviours are woven throughout the programme and at all institutional levels can more effectively nurture humanism and professionalism in its trainees" [2].

\section{RERERENCES}

1. NOVA Online:The Hipocratic Oath www.pbs.org/wgbh/nova/doctors/oathclassical.html

2. Markakis KM, Beckman HB, Suchman AL and Frankel RM. Cultivating Humanistic Values and Attitudes in Residency Training. Academic Medicine 2000; 75: 141-149

3. Rosenberg W and Donald A. Evidence based medicine: an approach to clinical problem-solving. BMJ.1995; 310:1122-1126

4. Justin RG .Can a Physician Always Be Compassionate? Hastings Center Report 30, No. 4; 2000: 26-27

5. Mayer ML .Simple Gestures. Obstetrics \& Gynecology .Vol.103, No. 1, January 2004: 3-4

6. Brody H. The Family Physician: What Sort of Person? Fam Med 1998; 30(8): 589-593

7. Martin AR. Stress in residency: a challenge to personal growth. J Gen Intern Med. 1986; JulAug; 1 (4) 252-257

8. Buysse DJ, Barzansky B, Dinges D, Hogan E, Hunt CE, Owens J, et al. Sleep, fatigue, and medical training: setting an agenda for optimal learning and patient care. Sleep 2003;26:218-225.

9. Adebamowo CA, Ezeome ER, Ajuwon AJ and Adekunle OO. Job stress associated with surgical training in Nigeria. Afr. J. Med. med. Sci. 1998; Sep-Dec; 27(3-4): 233-237.

10. Ndom RJ and Makanjuola AB. Perceived stress factors among resident doctors in a Nigerian 
teaching hosital.West Afr J Med. 2004; Jul-Sep; 1995; 123: 512-517

23(3): 232-235

11. Smith DG, and Newton L. Physician and patient: respect for mutuality. Theor. Med 1984; 5 : 43-60

12. Green, MJ. What (if anything) is wrong with residency overwork. Annals of Internal Medicine
13. Talabi OA. A questionnaire survey of senior house officers/registrars response to their training at University College Hospital, Ibadan. West Afr. J Med. 2003; Jun 22(2): 161-163

14. Barondess JA. Is Osler Dead? Perspectives in Biology and Medicine. 2002; 4(1): 65-84 